PROCEEDINGS OF THE

AMERICAN MATHEMATICAL SOCIETY

Volume 134, Number 9, September 2006, Pages 2549-2558

S 0002-9939(06)08172-X

Article electronically published on April 7, 2006

\title{
A MATRICIAL CORONA THEOREM
}

\author{
TAVAN TRENT AND XINJUN ZHANG
}

(Communicated by Joseph A. Ball)

\begin{abstract}
We show that a usual corona-type theorem on a space of functions automatically extends to a matrix version.
\end{abstract}

In this paper we give a simple algebraic argument to extend the corona theorem for a given algebra of bounded functions to a one-sided infinite matrix corona theorem. In addition, we provide estimates for the size of solutions. For the algebra $H^{\infty}(D)$, this was established by Fuhrmann 5 for the finite matrix case and by Vasyunin (see Nikolski [7) for the one-sided infinite case. We note that an important result of Treil [11] shows that a complete extension of the corona theorem to two-sided infinite matrices is not possible, in general. Our technique is similar to that of Fuhrmann [5], but our proofs are different. We prove a general version which can be applied to such algebras as the multipliers on Dirichlet space. Moreover, there is an $H^{p}$ version for $H^{\infty}\left(D^{n}\right)$. Our results provide sharper estimates than those obtained by one of the authors, Zhang [15].

For ease of notation, we will assume that $\mathcal{A}$ denotes a multiplier algebra for a reproducing kernel Hilbert space of functions on $\Omega$. That is, $H(\Omega)$ is a Hilbert space of functions on $\Omega$, such that, for each $w \in \Omega$, there exists a unique $k_{w} \in H(\Omega)$, the reproducing kernel, which satisfies $f(w)=\left\langle f, k_{w}\right\rangle_{H(\Omega)}$ for all $f \in H(\Omega)$. Then $\mathcal{A}=\left\{g \in H(\Omega) \mid M_{g} \in B(H(\Omega))\right.$, where $\left(M_{g} f\right)(w)=g(w) f(w)$ for all $\left.w \in \Omega\right\}$. If $B$ is an algebra of bounded functions, which is not such a multiplier algebra, we only consider finite matrices, so the arguments appearing below are easily modified in this case.

For $\left\{f_{j}\right\}_{j=1}^{\infty} \subset \mathcal{A}$ and $z \in \Omega$, we let $F(z)=\left(f_{1}(z), f_{2}(z), \ldots\right)$ and define $M_{F}\left(\left\{g_{j}\right\}_{j=1}^{\infty}\right)=\sum_{j=1}^{\infty} f_{j} g_{j}$, an operator acting from $\bigoplus_{1}^{\infty} H(\Omega)$ to $H(\Omega) . M_{F}^{T}$ will denote the transpose of $M_{F}$ acting from $H(\Omega)$ to $\bigoplus_{1}^{\infty} H(\Omega)$.

Since $M_{F}^{*}\left(k_{w}\right)=\left(f_{1} \overline{(w)} k_{w}, f_{2} \overline{(w)} k_{w}, \ldots\right)^{T}$, we see that

$$
\sum_{j=1}^{\infty}\left|f_{j}(w)\right|^{2} \leq\left\|M_{F}\right\|^{2} \text { for all } w \in \Omega .
$$

Received by the editors September 8, 2004 and, in revised form, January 13, 2005.

2000 Mathematics Subject Classification. Primary 32A65, 46J20.

Key words and phrases. Matrix corona theorem.

This work was partially supported by NSF Grant DMS-0400307.

(C)2006 American Mathematical Society Reverts to public domain 28 years from publication 
We assume that $\mathcal{A}$ satisfies a "corona theorem"; that is,

Theorem (CT). Assume that $\left\{f_{j}\right\}_{j=1}^{\infty} \subset \mathcal{A}$ and $F=\left(f_{1}, f_{2}, \ldots\right)$ satisfies

$$
\text { (1) } 0<\delta^{2} \leq F(z) F(z)^{*}
$$

and

$$
\text { (2) } \max \left\{\left\|M_{F}\right\|,\left\|M_{F}^{T}\right\|\right\}=1 \text {. }
$$

Then there exists $\left\{g_{j}\right\}_{j=1}^{\infty} \subset \mathcal{A}$, such that if $G=\left(g_{1}, g_{2}, \ldots\right)$,

(a) $F G^{T}=1$ in $\Omega$,

(b) $\max \left\{\left\|M_{G}\right\|,\left\|M_{G}^{T}\right\|\right\}<\infty$.

When CT holds for $\mathcal{A}$ and $F$ satisfies (1) and (2), we define

$$
C_{1}(\delta)=\inf \left\{\max \left\{\left\|M_{G}\right\|,\left\|M_{G}^{T}\right\|\right\}: F G^{T}=1 \text { and } G=\left(g_{1}, \ldots\right) \text { with } g_{i} \in \mathcal{A}\right\} \text {. }
$$

Note. $\quad$ (i) In some algebras such as multipliers on Dirichlet space

$$
\left\|M_{G}^{T}\right\|<\infty \Rightarrow\left\|M_{G}\right\|<\infty
$$

so (2) above may be replaced by

$$
\left(2^{\prime}\right)\left\|M_{F}^{T}\right\|=1 .
$$

(See Trent [12].)

(ii) For $\mathcal{A}=H^{\infty}(\Omega), \Omega \subset \mathbb{C}^{n}$, bounded and open,

$$
\left\|M_{F}\right\|=\left\|M_{F}^{T}\right\|=\sup _{z \in \Omega}\left(\sum_{j=1}^{\infty}\left|f_{j}(z)\right|^{2}\right)^{\frac{1}{2}} .
$$

So (2) above is equivalent to $\left(2^{\prime}\right) \sup _{z \in \Omega}\left(\sum_{j=1}^{\infty}\left|f_{j}(z)\right|^{2}\right)=1$.

(iii) For $F=\left(f_{1}, \ldots, f_{j}\right)$, a finite number of elements of $\mathcal{A},(2)$ may be omitted from the hypotheses.

[From (i), (ii), and (iii), we see that variations of CT involving changing hypothesis (2) are possible. Extensions of CT for such versions follow from our techniques below, but we will stick to the above formulation of CT.]

We establish the "matricial corona theorem" for such algebras $\mathcal{A}$, which satisfy CT.

Theorem (MCT). Assume that $\boldsymbol{C T}$ holds for $\mathcal{A}$. Let $F$ denote an $m \times \infty$ matrix of elements of $\mathcal{A}$ satisfying:

$$
\text { (1) } 0<\epsilon^{2} I_{m} \leq F(z) F(z)^{*} \text { for all } z \in \Omega
$$

and

$$
\text { (2) } \max \left\{\left\|M_{F}\right\|,\left\|M_{F}^{T}\right\|\right\}=1 \text {. }
$$

Then there exists an $m \times \infty$ matrix $G$ with entries in $\mathcal{A}$ satisfying

$$
\begin{aligned}
& \text { (a) } F G^{T}=I_{m} \text {, } \\
& \text { (b) } \max \left\{\left\|M_{G}\right\|,\left\|M_{G}^{T}\right\|\right\}<\infty
\end{aligned}
$$


Moreover, we may choose $G$, as above, so that

$$
\max \left\{\left\|M_{G}\right\|,\left\|M_{G}^{T}\right\|\right\} \leq \sqrt{m} m ! C_{1}\left(\frac{\epsilon^{2 m}}{m !}\right) .
$$

Denote the rows of $F$ satisfying (1) and (2) of MCT by $f_{1}, \ldots, f_{m}$. For $1 \leq j \leq$ $m$, we will denote the $j$ th term of the Koszul complex with 0 th element $f_{p}$ by $Q_{p}^{(j)}$. This means that for each $z \in \Omega$

$$
\begin{aligned}
\operatorname{ker} f_{p}(z) & \supseteq \operatorname{ran} Q_{p}^{(1)}(z), \\
\operatorname{ker} Q_{p}^{(1)}(z) & \supseteq \operatorname{ran} Q_{p}^{(2)}(z)
\end{aligned}
$$

and, moreover,

$$
\begin{aligned}
f_{p}(z)^{*} f_{p}(z)+Q_{p}^{(1)}(z) Q_{p}^{(1)}(z)^{*} & =\left(f_{p}(z) f_{p}(z)^{*}\right) I, \\
Q_{p}^{(1)}(z)^{*} Q_{p}^{(1)}(z)+Q_{p}^{(2)}(z) Q_{p}^{(2)}(z)^{*} & =\left(f_{p}(z) f_{p}(z)^{*}\right) I
\end{aligned}
$$

Since at each $z \in \Omega, f_{p}(z) f_{p}(z)^{*} \geq \epsilon^{2}>0$, we deduce that

$$
\begin{aligned}
\operatorname{ker} f_{p}(z) & =\operatorname{ran} Q_{p}^{(1)}(z), \\
\operatorname{ker} Q_{p}^{(1)}(z) & =\operatorname{ran} Q_{p}^{(2)}(z)
\end{aligned}
$$

In addition,

$$
Q_{p}^{(j)}(z) Q_{q}^{(j+1)}(z)=-Q_{q}^{(j)}(z) Q_{p}^{(j+1)}(z)
$$

and

$$
f_{1}(z) Q_{2}^{(1)}(z) \cdots Q_{m}^{(m-1)}(z) Q_{m}^{(m-1)}(z)^{*} \cdots f_{1}(z)^{*}=\operatorname{det}\left(F(z) F(z)^{*}\right) .
$$

See the Appendix for the existence of $\left\{Q_{p}^{(j)}(z)\right\}_{j=1}^{\infty}$ and the above properties.

We will need a lemma.

Lemma. Let $A=\left(a_{1}, a_{2}, \ldots\right), a_{i} \in \mathcal{A}$, and assume that

$$
\max \left\{\left\|M_{A}\right\|,\left\|M_{A}^{T}\right\|\right\}<\infty .
$$

Fix $z \in \Omega$ and let

$$
Q^{(j)}(z)=Q_{A}^{(j)}(z)
$$

Then for $1 \leq j$

$$
\left\|M_{Q^{(j)}}\right\| \leq(j+1)\left\|M_{A}\right\| \text { and }\left\|M_{Q^{(j)}}^{T}\right\| \leq(j+1)\left\|M_{A}^{T}\right\| .
$$

Proof. We will show that the first inequality holds. The second follows in a similar manner. See the Appendix for notation. Let $\mathcal{I}_{j}$ denote increasing $j$-tuples of 
positive integers. Now

$$
\begin{aligned}
Q^{(j)}(z)^{*} \underline{x}(z) & =\overline{A(z)} \wedge \underline{x}(z) \\
& =\left(\sum_{j=1}^{\infty} \overline{a_{j}(z)} e_{j}\right) \wedge\left(\sum_{\pi \in \mathcal{I}_{j}} x_{\pi}(z) e_{\pi}\right) \\
& =\sum_{j=1}^{\infty} \sum_{\pi \in \mathcal{I}_{j}} \overline{a_{j}(z)} x_{\pi}(z) e_{j} \wedge e_{\pi} \\
& =\sum_{1 \leq i_{1}<i_{2}<\cdots<i_{j+1}} \sum_{r=1}^{j+1}(-1)^{r-1} \overline{a_{r}(z)} x_{i_{1}, \ldots, \hat{i_{r}, \ldots, i_{j+1}}} e_{i_{1}, \ldots, i_{j+1}} .
\end{aligned}
$$

Thus

$$
\begin{aligned}
\left\|M_{Q^{(j)}}^{*} \underline{x}\right\|^{2} & =\sum_{\substack{\left.\sigma \in \mathcal{I}_{j+1} \\
\sigma=\left(i_{1}, \ldots, i_{j+1}\right)\right)}}\left\|\sum_{r=1}^{j+1}(-1)^{r-1} M a_{r}^{*} x_{\sigma-\left\{i_{r}\right\}}\right\|^{2} \\
\leq & (j+1) \sum_{i_{1}<\cdots<i_{j+1}} \sum_{r=1}^{j+1}\left\|M a_{i_{r}}^{*} x_{\sigma-\left\{i_{r}\right\}}\right\|^{2} \\
& =(j+1) \sum_{i_{1}<\cdots<i_{j+1}}\left(\left\|M a_{i_{1}}^{*} x_{\sigma-\left\{j_{1}\right\}}\right\|^{2}+\ldots\right. \\
& \leq(j+1)\left\|M_{A}^{*}\right\|^{2}\left(\sum_{i_{2}<\cdots<i_{j+1}}\left\|a_{i_{j+1}}^{*} x_{\sigma-\left\{i_{j+1}\right\}}\right\|^{2}\right) \\
& +\sum_{i_{1}<\cdots, i_{j+1}} \|^{2}+\ldots \\
\leq & \left.\left\|x_{i_{1}, \ldots, i_{j}}\right\|^{2}\right) \\
&
\end{aligned}
$$

So

$$
\left\|M_{Q^{(j)}}\right\| \leq(j+1)\left\|M_{A}\right\| .
$$

We are ready to begin the proof of $\mathbf{M C T}$.

Proof. (MCT) Since $\epsilon^{2} I_{m} \leq F(z) F(z)^{*}$ for $z \in \Omega$, we have $\epsilon^{2 m} \leq \operatorname{det}\left(F(z) F(z)^{*}\right)$ for all $z \in \Omega$. Thus, if we define

$$
H(z)=f_{1}(z) Q_{2}^{(1)}(z) \cdots Q_{m}^{(m-1)}(z), \text { then } H=\left(h_{1}, h_{2}, \ldots\right)
$$

with $h_{i} \in \mathcal{A}$ and

$$
\epsilon^{2 m} \leq \operatorname{det}\left(F(z) F(z)^{*}\right)=H(z) H(z)^{*}
$$


by (5). From the lemma, we have

$$
\begin{aligned}
\left\|M_{H}\right\| \leq\left\|M_{f_{1}}\right\|\left\|M_{Q_{2}^{(1)}}\right\| \cdots\left\|M_{Q_{m}(m-1)}\right\| & \leq\left\|M_{f_{1}}\right\|\left(2\left\|M_{f_{2}}\right\|\right) \cdots\left(m\left\|M_{f_{m}}\right\|\right) \\
& =m ! \prod_{j=1}^{m}\left\|M_{f_{j}}\right\| \\
& \leq m !\left\|M_{F}\right\|^{m} \\
& \leq m !, \text { since }\left\|M_{F}\right\| \leq 1 .
\end{aligned}
$$

Applying $\mathbf{C T}$ to $\frac{H}{m !}$ with $\delta=\frac{\epsilon^{m}}{m !}$, we get

$$
R=\left(g_{1}, g_{2}, \ldots\right) \text { with } g_{i} \in \mathcal{A}
$$

so that

$$
H R^{T}=1
$$

and

$$
\max \left\{\left\|M_{R}\right\|,\left\|M_{R}^{T}\right\|\right\} \leq C_{1}\left(\frac{\epsilon^{m}}{m !}\right) .
$$

Define

$$
\begin{aligned}
G^{T}= & {\left[Q_{2}^{(1)} \cdots Q_{m}^{(m-1)} R^{T},-Q_{1}^{(1)} Q_{3}^{(2)} \cdots Q_{m}^{(m-1)} R^{T}, \ldots,\right.} \\
& (-1)^{j-1} Q_{1}^{(1)} Q_{2}^{(2)} \cdots \widehat{Q_{j}^{(j)}} Q_{j+1}^{(j)} \cdots Q_{m}^{(m-1)} R^{T}, \ldots \\
& \left.(-1)^{m-1} Q_{1}^{(1)} \cdots Q_{m-1}^{(m-1)} R^{T}\right] .
\end{aligned}
$$

Then

$$
\left\|M_{G^{T}}\right\| \leq \sqrt{m} m ! C_{1}\left(\frac{\epsilon^{m}}{m !}\right)
$$

Similarly,

$$
\left\|M_{G}\right\| \leq \sqrt{m} m ! C_{1}\left(\frac{\epsilon^{m}}{m !}\right)
$$

We need only check that

$$
F G^{T}=I_{m}
$$

But

$$
f_{j} G^{T}=\left[f_{j} Q_{2}^{(1)} \cdots Q_{m}^{(m-1)} R^{T}, \ldots,(-1)^{m-1} f_{j} Q_{1}^{(1)} \cdots Q_{m-1}^{(m-1)} R^{T}\right] .
$$

The indices of the $Q$ 's in each term consist of $I_{p}=\{1, \ldots, m\}-\{p\}$ for $p=1, \ldots, m$. For $p \neq j$, we see that

$$
(*) \stackrel{\text { def }}{=} f_{j} Q_{1}^{(1)} \cdots Q_{p-1}^{(p-1)} Q_{p+1}^{(p)} \cdots Q_{m}^{(m-1)} R^{T}=0 .
$$

This follows since $f_{j} Q_{j}^{(1)}=0$ and $Q_{s}^{(r)} Q_{t}^{(r+1)}=-Q_{t}^{(r)} Q_{s}^{(r+1)}$, so since $j \in$ $\{1, \ldots, m\}-\{p\}$, we may "anti-" commute the $Q$ 's until we get

$$
(*)=f_{j} Q_{j}^{(1)} \cdots R^{T}=0 .
$$


Otherwise, $p=j$, and we have

$$
\begin{aligned}
& (-1)^{j-1} f_{j} Q_{1}^{(1)} \cdots Q_{j-1}^{(j-1)} Q_{j+1}^{(j)} \cdots Q_{m}^{(m-1)} R^{T} \\
= & (-1)^{j-2} f_{1} Q_{j}^{(1)} Q_{2}^{(2)} \cdots Q_{j-1}^{(j-1)} Q_{j+1}^{(j)} \cdots Q_{m}^{(m-1)} R^{T} \\
= & (-1)^{j-3} f_{1} Q_{2}^{(1)} Q_{j}^{(2)} Q_{3}^{(3)} \cdots Q_{j-1}^{(j-1)} Q_{j+1}^{(j)} \cdots Q_{m}^{(m-1)} R^{T} \\
& \vdots \\
= & f_{1} Q_{2}^{(1)} Q_{3}^{(2)} \cdots Q_{m}^{(m-1)} R^{T} \\
= & H R^{T} \\
= & 1 .
\end{aligned}
$$

This completes our proof of the matricial corona theorem.

Applications:

(1) For $\mathcal{A}=H^{\infty}(D)$, the finite corona theorem is due to Carleson [2] and the infinite corona theorem is due to Rosenblum [8] and Tolokonnikov [10, independently. Our results give the matricial corona theorem due to Fuhrmann and Vasyunin. In this case, our conditions $(*)$ show that if $\epsilon^{2} I_{m} \leq F(z) F(z)^{*} \leq I_{m}$, then $\left\|M_{Q_{p}^{(i)}}\right\| \leq 1$. Therefore, if $F$ is an $m \times \infty$ matrix with entries $f_{i j} \in H^{\infty}(D)$ satisfying $\delta^{2} I_{m} \leq F(z) F(z)^{*} \leq I_{m}$ for all $z \in D$, we get an $\infty \times m$ solution $G$ with entries $f_{j} \in H^{\infty}(D)$ satisfying

$$
\left\|M_{G}\right\|=\left\|M_{G}^{T}\right\| \leq \sqrt{m} C_{1}\left(\delta^{m}\right) .
$$

For $\delta \approx 0$, the best estimate of $C_{1}(\delta)$ is $\frac{C_{0}}{\delta^{2}} \ln \frac{1}{\delta}$ due to Uchiyama (see Nikolski (7]).

So we only get

$$
\left\|M_{G}\right\|=\left\|M_{G}^{T}\right\| \leq \sqrt{m} \frac{C_{0}}{\delta^{2 m}} \log \frac{1}{\delta^{m}} .
$$

We note that for this particular case, where $\mathcal{A}=H^{\infty}(D)$, a better estimate in the matricial corona theorem due to Trent [13] gives

$$
\left\|M_{G}\right\| \leq \frac{C_{0}}{\delta^{m+1}} \log \frac{1}{\delta^{m}} .
$$

(2) If $\Omega \subset \mathbb{C}$ is a finitely connected domain, then the corona theorem holds for $H^{\infty}(\Omega)$ by Stout [9] and Forelli [4]. (See Fisher [3] for an exposition of these results.) Our result gives a matricial version.

(3) For $\mathcal{A}=\mathcal{D}^{2}(D) \cap H^{\infty}(D)$ (where $\mathcal{D}^{2}(D)$ denotes Dirichlet space), by results of Nicolau [6], $\mathcal{A}$ satisfies a "finite" corona theorem. Thus our results give a "finite" matricial version corona theorem for $\mathcal{D}^{2}(D) \cap H^{\infty}(D)$.

(4) For $\mathcal{A}=\mathcal{M}\left(\mathcal{D}^{2}(D)\right)$, multipliers on $\mathcal{D}^{2}(D)$, the $\infty$-corona theorem is due to Trent [12]. Our results give a matricial corona theorem for $\mathcal{M}\left(D^{2}(D)\right)$.

(5) For $1 \leq p<\infty$, let

$$
\begin{aligned}
& \mathcal{H}^{p}\left(D^{n}\right)=\left\{f: D^{n} \rightarrow l^{2} \mid f \text { is analytic, } l^{2} \text {-valued in } D^{n}\right. \text { and } \\
& \left.\quad|f|_{p}^{p} \stackrel{\text { def }}{=} \sup _{r / 1}\left(\int_{T^{n}}\left\|f\left(r e^{i t_{1}}, r e^{i t_{2}}, \ldots, r e^{i t_{n}}\right)\right\|_{2}^{p} d \sigma\left(t_{1}\right) \cdots d \sigma\left(t_{n}\right)<\infty\right)\right\} .
\end{aligned}
$$

In [14, Trent has proven the $\mathcal{H}^{p}$-corona theorem. 
Theorem (Hי-CT). Let $F \in \mathcal{H}^{\infty}\left(D^{n}\right)$ satisfy $0<\epsilon^{2} \leq F(z) F(z)^{*} \leq 1$ for all $z \in D^{n}$. Then the Toeplitz operator, $T_{F}: \mathcal{H}^{p}\left(D^{n}\right) \rightarrow H^{p}\left(D^{n}\right)$, is onto for each $1 \leq p<\infty$.

[Note: The case that $p=\infty$ is an unsolved problem for the polydisk.]

A modification of the proof of MCT leads to an $H^{p}$-matricial corona theorem. That is,

Theorem (Hי-MCT). Let $F$ be a $m \times \infty$ matrix of elements of $H^{\infty}\left(D^{n}\right)$. Assume that

$$
0<\epsilon^{2} I_{m} \leq F(z) F(z)^{*} \leq I_{m} .
$$

Then for $1 \leq p<\infty$, the Toeplitz operator $T_{F}: \mathcal{H}^{p}\left(D^{n}\right) \rightarrow \bigoplus_{1}^{m} H^{p}\left(D^{n}\right)$, is onto.

Proof. We will outline the modifications of the proof of the MCT necessary for this proof. First

by hypothesis. For

$$
\left\|T_{F}\right\|=\sup _{z \in D^{n}}\|F(z)\|_{B\left(l^{2}, \mathbb{C}^{m}\right)} \leq 1
$$

with $z \in D^{n}$, we have

$$
H(z)=f_{1}(z) Q_{f_{2}}^{(1)}(z) \cdots Q_{f_{m}}^{(m-1)}(z)
$$

$$
\epsilon^{2 m} \leq H(z) H(z)^{*} \leq 1 .
$$

This follows since

$$
Q_{f_{j}}^{(r)}(z) Q_{f_{j}}^{(r)}(z)^{*} \leq\left(f_{j}(z) f_{j}(z)^{*}\right) I \leq I .
$$

Thus $T_{H}: \mathcal{H}^{p}\left(D^{n}\right) \rightarrow H^{p}\left(D^{n}\right)$ is onto. Let $\underline{h}=\left(h_{1}, \ldots, h_{m}\right) \in \bigoplus_{1}^{m} H^{p}\left(D^{n}\right)$. Choose $u_{k} \in H^{p}\left(D^{n}\right)$, so that $T_{H}\left(u_{k}\right)=H u_{k}=h_{k}$ for $k=1, \ldots, m$. Let

$$
\underline{g}^{T}=\left[Q_{2}^{(1)} \cdots Q_{m}^{(m-1)} u_{1}, \ldots,(-1)^{m-1} Q_{1}^{(1)} \cdots Q_{m-1}^{(m-1)} u_{m}\right] .
$$

Then

$$
F \underline{g}=\underline{h} .
$$

Moreover, $\underline{g}$ can be chosen so that

$$
\begin{aligned}
\|\underline{g}\|_{p}^{p} & \leq \sum_{j=1}^{m}\left\|u_{j}\right\|_{p}^{p} \\
& \leq\|\underline{h}\|_{p}^{p} \\
& \leq m\left\|\left[\left.T_{H}\right|_{\left(\operatorname{ker} T_{H}\right)^{\perp}}\right]^{-1}\right\|^{p}\|h\|_{p}^{p} .
\end{aligned}
$$

Finally,

Here $C_{1}(\delta, p)$ denotes

$$
\|g\|_{p} \leq m^{\frac{1}{p}} C_{1}\left(\epsilon^{m}, p\right)\|h\|_{p}
$$

$$
\left\|\left(\left.T_{F}\right|_{\left(\operatorname{ker} T_{F}\right)^{\perp}}\right)^{-1}\right\|_{B\left(H^{p}\left(D^{n}\right), \mathcal{H}^{p}\left(D^{n}\right)\right)} .
$$

The estimate for $C_{1}(\delta, p)$ in [14] is

So

$$
C_{1}(\delta, p) \leq \frac{C_{0} p^{2 n-1}}{\delta^{3 n+1}} .
$$

$$
C_{m}(\delta, p) \leq \frac{m^{\frac{1}{p}} C_{0} p^{2 n-1}}{\delta^{(3 n+1) m}}
$$

gives the matricial estimate. 


\section{Appendix}

Let $\mathcal{A}$ be an algebra of functions defined on $\Omega$. The basic exterior algebra proof of the linear algebra results we need concerning the Koszul complex can be found in, for example, Birkhoff-Mac Lane [1, Problem 4, page 566].

We will sketch the basic idea. Note that although our operators defined below are (of course) "bases free", it is only with respect to a particular choice of basis that the entries of the corresponding matrices belong to $\mathcal{A}$.

For our notation, $l_{(n)}^{2}$ will denote the exterior product of $l^{2}$ with itself $n$-times, i.e. $\quad l_{(n)}^{2}=l^{2} \wedge \cdots \wedge l^{2}(n$ times $)$. For $n=0, l_{(0)}^{2}=\mathbb{C}$. Let $\left\{e_{j}\right\}_{j=1}^{\infty}$ denote the standard basis in $l^{2}$. If $\mathcal{I}_{n}$ denotes increasing $n$-tuples of positive integers and if $\left(i_{1}, \ldots, i_{n}\right) \in \mathcal{I}_{n}$, we let $\pi_{n}=\left\{i_{1}, \ldots, i_{n}\right\}$, and abusing notation, we write $\pi_{n} \in \mathcal{I}_{n}$. $l_{(n)}^{2}$

Define $e_{\pi_{n}}=e_{i_{1}} \wedge \cdots \wedge e_{i_{n}}$. Then $\left\{e_{\pi_{n}}\right\}_{\pi_{n} \in \mathcal{I}_{n}}$ denotes the standard basis for

For $f_{j} \sim\left\{v_{n}\right\}_{n=1}^{\infty}$ and $v_{n} \in \mathcal{A}$, we assume that $\epsilon^{2} \leq f_{j}(\mathbf{z}) f_{j}(\mathbf{z})^{*} \leq 1$ for all $\mathbf{z} \in \Omega$. Fix $\mathbf{z} \in \Omega$. For $n=0,1, \ldots$ define

$$
Q_{j}^{(n) *}(\mathbf{z}): l_{(n)}^{2} \rightarrow l_{(n+1)}^{2}
$$

by

$$
Q_{j}^{(n) *}(\mathbf{z})\left(w_{n}\right)=\overline{f_{j}(\mathbf{z})} \wedge w_{n}, \text { where } w_{n} \in l_{(n)}^{2} .
$$

Clearly (1) holds and since

$$
Q_{j}^{(n+1) *}(\mathbf{z}) Q_{k}^{(n) *}(\mathbf{z})\left(w_{n}\right)=\overline{f_{j}(\mathbf{z})} \wedge \overline{f_{k}(\mathbf{z})} \wedge w_{n}=-Q_{k}^{(n+1) *}(\mathbf{z}) Q_{j}^{(n) *}(\mathbf{z})\left(w_{n}\right)
$$

we see that (4) holds.

Now

$$
Q_{j}^{(n) *}(\mathbf{z})\left(e_{\pi_{n}}\right)=\sum_{p=1}^{\infty} \overline{v_{p}(\mathbf{z})} e_{p} \wedge e_{\pi_{n}}
$$

so with respect to the standard basis, entries of $Q_{j}^{(n) *}(\mathbf{z})$ are 0 or else $\pm \overline{v_{n}(\mathbf{z})}$ for some $n$. Thus $Q^{(n)}(\cdot)$ has entries belonging to $\mathcal{A}$ with respect to the standard basis.

Proof. Fix $\mathbf{z} \in \Omega$ and let $a=\overline{f_{j}(\mathbf{z})}$, for fixed $j$, and $Q_{n}=Q_{j}^{(n)}(\mathbf{z})$. Then $Q_{n}^{*}\left(w_{n}\right)=$ $a \wedge w_{n}$. Choose an orthonormal basis $\left\{u_{n}\right\}_{n=1}^{\infty}$ of $l^{2}$ with $u_{1}=\frac{a}{\|a\|}$. (Note that $\|a\|^{2} \geq \epsilon^{2}$.) Then it follows that for $\pi_{n} \in \mathcal{I}_{n}$ and $u_{\pi_{n}}=u_{i_{1}} \wedge \cdots \wedge u_{i_{n}}$, we have that $\left\{u_{\pi_{n}}\right\}_{\pi_{n} \in \mathcal{I}_{n}}$ is an orthonormal basis for $l_{(n)}^{2}$.

Thus

$$
\begin{aligned}
Q_{n}\left(w_{n+1}\right) & =\sum_{\pi_{n} \in I_{n}}\left\langle Q_{n}\left(w_{n+1}\right), u_{\pi_{n}}\right\rangle u_{\pi_{n}} \\
& =\sum_{\pi_{n} \in \mathcal{I}_{n}}\left\langle w_{n+1}, a \wedge u_{\pi_{n}}\right\rangle u_{\pi_{n}} \\
& =\|a\| \sum_{\pi_{n} \in \mathcal{I}_{n}}\left\langle w_{n+1}, u_{1} \wedge u_{\pi_{n}}\right\rangle u_{\pi_{n}} .
\end{aligned}
$$

We wish to show that for $n=0,1, \ldots$,

$$
Q_{n}^{*} Q_{n}+Q_{n+1} Q_{n+1}^{*}=\|a\|^{2} I_{l_{(n+1)}^{2}} .
$$


For $n=0, \frac{Q_{0}^{*} Q_{0}}{\|a\|^{2}}$ is the rank one projection of $l^{2}$ onto $a$. So given (7), $\frac{Q_{1} Q_{1}^{*}}{\|a\|^{2}}$ is a projection. But then $\frac{Q_{1}^{*} Q_{1}}{\|a\|^{2}}$ must be a projection. Applying (7) again, we see that $\frac{Q_{2} Q_{2}^{*}}{\|a\|^{2}}$ is a projection. Repeating this procedure, we conclude that $\frac{Q_{n} Q_{n}^{*}}{\|a\|^{2}}$ is the projection onto the range of $Q_{n}$. Also, given (7), it follows that $\operatorname{Ker} Q_{n}=\operatorname{ran}$ $Q_{n+1}$.

To prove (7) it suffices to check that for $w_{n+1} \in l_{(n+1)}^{2}$,

$$
\left\|Q_{n}\left(w_{n+1}\right)\right\|^{2}+\left\|Q_{n+1}^{*}\left(w_{n+1}\right)\right\|^{2}=\|a\|^{2}\left\|w_{n+1}\right\|^{2} .
$$

Denote $w_{n+1}$ by $w$. Then from (8), we see that

$$
\begin{aligned}
\left\|Q_{n}(w)\right\|^{2} & =\|a\|^{2} \sum_{\substack{\pi_{n} \in \mathcal{I}_{n} \\
1 \notin \pi_{n}}}\left|\left\langle w, u_{1, \pi_{n}}\right\rangle\right|^{2} \\
& =\|a\|^{2} \sum_{\substack{\pi_{n+1} \in \mathcal{I}_{n+1} \\
1 \in \pi_{n+1}}}\left|\left\langle w, u_{\pi_{n+1}}\right\rangle\right|^{2} .
\end{aligned}
$$

Also, since

$$
\begin{aligned}
Q_{n+1}^{*}(w)=a \wedge \underline{w} & =\|a\| u_{1} \wedge \sum_{\pi_{n+1} \in \mathcal{I}_{n+1}}\left\langle w, u_{\pi_{n+1}}\right\rangle u_{\pi_{n+1}} \\
& =\|a\| \sum_{\substack{\pi_{n+1} \in \mathcal{I}_{n+1} \\
1 \notin \pi_{n+1}}}\left\langle w, u_{\pi_{n+1}}\right\rangle u_{1} \wedge u_{\pi_{n+1}},
\end{aligned}
$$

we compute that

$$
\left\|Q_{n+1}^{*}(w)\right\|^{2}=\|a\|^{2} \sum_{\substack{\pi_{n+1} \in \mathcal{I}_{n+1} \\ 1 \notin \pi_{n+1}}}\left|\left\langle w, u_{\pi_{n+1}}\right\rangle\right|^{2} .
$$

So (7) holds.

Let $a=f_{j}(\mathbf{z})$ and $b=f_{k}(\mathbf{z})$ with $j \neq k$. Then

$$
b=\langle b, a\rangle \frac{a}{\|a\|^{2}}+b_{\perp}, \text { where } \quad b_{\perp} \perp a .
$$

We claim that $Q_{b}^{(n) *} Q_{a}^{(n)}+Q_{a}^{(n+1)} Q_{b}^{(n+1) *}=\langle a, b\rangle I$.

If $b$ is replaced by $b=\langle b, a\rangle \frac{a}{\|a\|^{2}}$, this follows from (7). So we need only check that, if $c \perp a$ in $l^{2}$, then

$$
Q_{c}^{(n) *} Q_{a}^{(n)}+Q_{a}^{(n+1)} Q_{c}^{(n+1) *}=0 .
$$

This easily follows by choosing an orthonormal basis for $l^{2},\left\{u_{j}\right\}_{j=1}^{\infty}$ with $u_{1}=\frac{a}{\|a\|}$ and $u_{2}=\frac{c}{\|c\|}$.

For $k=2, \ldots, m$, let $a_{k}{ }^{\prime}=P_{s p\left\{a_{1}, \ldots, a_{k-1}\right\}}^{\perp}\left(a_{k}\right)$. Then, using (7) and (4) repeatedly, we see that for $1 \leq r \leq m$ and $\mathbf{z} \in \Omega$ fixed,

$$
a_{1} Q_{a_{2}}^{(1)} \ldots Q_{a_{r}}^{(r-1)} Q_{a_{r}}^{(r-1) *} \ldots a_{1}^{*}=\left\|a_{1}\right\|^{2} \prod_{j=2}^{r}\left\|a_{j}^{\prime}\right\|^{2} .
$$


So when $r=m,(5)$ holds. That is,

$$
a_{1} Q_{a_{2}}^{(1)} \ldots Q_{a_{m}}^{(m-1)} Q_{a_{m}}^{(m-1) *} \ldots a_{1}^{*}=\left\|a_{1}\right\|^{2} \prod_{j=2}^{m}\left\|a_{j}^{\prime}\right\|^{2}=\operatorname{det}\left(F(\mathbf{z}) F(\mathbf{z})^{*}\right) .
$$

This completes the proof of the linear algebra material.

\section{REFERENCES}

[1] G. Birkhoff and S. Mac Lane, Algebra, MacMillan, Toronto, 1971.

[2] L. Carleson, Interpolation by bounded analytic functions and the corona problem, Annals of Math. 76 (1962), 547-559. MR0141789 (25:5186)

[3] S. Fisher, Function Theory on Planar Domains, a Second Course in Complex Analysis, John Wiley and Sons, New York, 1983. MR0694693 (85d:30001)

[4] F. Forelli, Bounded holomorphic functions and projections, Illinois J. Math. 10 (1966), 367380. MR.0193534(33:1754)

[5] P. A. Fuhrmann, On the corona theorem and its applications to spectral problems in Hilbert space, Trans. Amer. Math. Soc. 132 (1968), 55-66. MR0222701 (36:5751)

[6] A. Nicolau, The corona property for bounded analytic functions in some Besov spaces, Proc. Amer. Math. Soc. 110 (1990), 135-140. MR.1017007 (90m:46090)

[7] N. K. Nikolski, Treatise on the Shift Operator, Springer-Verlag, New York, 1985. MR0827223 (87i:47042)

[8] M. Rosenblum, A corona theorem for countably many functions, Integral Equa. Oper. Theory 3 (1980), 125-137. MR0570865 (81e:46034)

[9] E. L. Stout, Bounded holomorphic functions on finite Riemann surfaces, Trans. Amer. Math. Soc. 120 (1965), 255-285. MR0183882 (32:1358)

[10] V. A. Tolokonnikov, Estimates in Carleson's corona theorem and finitely generated ideals in the algebra $H^{\infty}(D)$, Functional Anal. I Prilozhen 14 (1980), 85-86 (in Russian). MR0595742 (82a:46058)

[11] S. R. Treil, Angles between coinvariant subspaces and an operator-valued corona problem, a question of Szökefalvi-Nagy, Soviet Math. Dokl. 38 (1989), 394-399. MR0981054|(90b:47057)

[12] T. T. Trent, A corona theorem for multipliers on Dirichlet space, Integral Equa. Oper. Theory 49 (2004), 123-139. MR2057771

[13] _ A new estimate for the vector-valued corona problem, J. Func. Anal. 189 (2002), 267-282. MR:1887635 (2002m:30067)

[14] _ An $H^{p}$-corona theorem on the bidisk for infinitely many functions, submitted.

[15] X. Zhang, A matrix version of corona theorem for algebras of functions on reproducing kernel Hilbert spaces, Ph.D. dissertation, The University of Alabama, Tuscaloosa, AL, August 2004.

Department of Mathematics, The University of Alabama, Box 870350, Tuscaloosa, Alabama 35487-0350

E-mail address: ttrent@gp.as.ua.edu

Department of Mathematics, The University of Alabama, Box 870350, Tuscaloosa, Alabama 35487-0350

E-mail address: zhang010@bama.ua.edu 